Revue d'histoire de l'enfance " irrégulière »

Le Temps de l'histoire

$20 \mid 2018$

Sexualités juvéniles

\title{
La sexualité infantile et juvénile dans l'œuvre d'Auguste Forel. Formes pathologiques et propositions eugénistes
}

The Childhood and adolescent sexuality in the work of Auguste Forel.

Pathological Forms and Eugenics Proposals

Giovanni Cerro

(2) OpenEdition

Journals

Édition électronique

URL : http://journals.openedition.org/rhei/4177

DOI : 10.4000/rhei.4177

ISSN : $1777-540 \mathrm{X}$

Éditeur

Presses universitaires de Rennes

Édition imprimée

Date de publication : 15 novembre 2018

Pagination : $39-64$

ISBN : 978-2-7535-7571-4

ISSN : $1287-2431$

\section{Référence électronique}

Giovanni Cerro, « La sexualité infantile et juvénile dans l'œuvre d'Auguste Forel. Formes pathologiques et propositions eugénistes », Revue d'histoire de l'enfance « irrégulière » [En ligne], 20 | 2018, mis en ligne le 15 novembre 2020, consulté le 03 décembre 2020. URL : http://journals.openedition.org/rhei/4177 DOI : https://doi.org/10.4000/rhei.4177 


\section{La sexualité infantile et juvénile dans l'œuvre d'Auguste Forel}

\section{Formes pathologiques et propositions eugénistes}

L'objet de l'article est la question de la sexualité infantile et juvénile dans l'œuvre du savant suisse Auguste Forel (1848-1931), un des psychiatres européens le plus influent entre le $\mathrm{xIX}^{\mathrm{e}}$ et le $\mathrm{xx}^{\mathrm{e}}$ siècle. Son ouvrage de 1905 Die sexuelle Frage est un jalon dans l'histoire de la sexologie, bientôt traduit en français, italien et anglais. L'analyse de Forel regarde deux aspects qui sont deux côtés de la même médaille : d'une part, la reconstruction des stades du développement sexuel sain et normal; d'autre part, la tentative d'identifier et éventuellement corriger les principaux phénomènes pathologiques et les perversions sexuelles, comme la masturbation, l'homosexualité et la prostitution. Sa recherche est ainsi un point de convergence entre les approches psychiatriques des déviances sexuelles du XIX $\mathrm{X}^{\mathrm{e}}$ siècle, surtout la psychopathologie de Richard von Krafft-Ebing, et l'émergence d'un intérêt croissant pour la sexualité infantile, qui en partie précède la systématisation de Freud. Parallèlement à cette intention diagnostique, Forel développe un original projet eugéniste des réformes morales, sexuelles et pédagogiques, dans une perspective socialiste fortement critique du système capitaliste.

The focus of this paper is the discussion of the childhood and adolescent sexuality in the work of the Swiss scholar Auguste Forel (1848-1931), one of the most influent European psychiatrists between the XIXth and the XXth century. His 1905 study Die sexuelle Frage is a cornerstone in the history of sexology, soon translated in French, Italian, and English. Forel's examination concerns two features, like two sides of the same coin: on the one hand, the reconstruction of the stages of healthy sexual development; on the other hand, the attempt to identify and eventually correct the main pathological phenomena and sexual perversions, such as masturbation, homosexuality, and prostitution. Thus, his research is a point of convergence between the XIXth century psychiatric approaches to sexual deviance, Richard von Krafft-Ebing's psychopathology above all, and the emergence of a growing interest in childhood sexuality, which partly precedes Freud's systematization. Alongside this diagnostic intent, Forel develops an original set of moral, sexual and pedagogical reforms, within a socialist perspective that lead a strong critic of the capitalist system.

Mots-clés : Auguste Forel, sexualité, enfance, eugénisme.

Keywords: Auguste Forel, sexuality, childhood, eugenics.

\author{
Giovanni Cerro \\ Docteur en \\ philosophie, \\ Fondazione Collegio \\ San Carlo, Modena \\ (Italie)
}


1. Cf. Schaffner Anna Katharina, Modernism and Perversion: Sexual Deviance in Sexology and Literature, 1850-1930 [Modernisme et perversion : la déviance sexuelle dans la sexologie et dans la littérature, 1850-1930], Basingstoke, Palgrave Macmillan, 2012, $315 p$

2. Davidson Arnold I., L'émergence de la sexualité : épistémologie historique et formation des concepts (2001), Paris, Albin Michel, 2005, 365 p., en particulier le premier chapitre qui, dès son titre (Refermer les cadavres, p. 25-75) fait explicitement allusion au chapitre huit de la Naissance de la clinique. Une archéologie du regard médical de Foucault (Paris, PUF, 1963) intitulé Ouvrez quelques cadavres et consacré à la conception de l'anatomiste et physiologue Marie-François-Xavier Bichat en matière d'anatomie pathologique.

3. Cf. HaCking lan, Historical Ontology [Ontologie historique], Cambridge (Mass.), Harvard University Press, 2002, $279 \mathrm{p}$
$\mathrm{D}$ ans ses recherches sur la sexualité infantile et juvénile, un thème qui a été au cour de ses intérêts durant toute sa vie, le psychiatre suisse Auguste Forel (1848-1931) s'occupe en premier lieu du développement dit " normal " qui doit aboutir à la maturité sexuelle, atteinte selon lui chez les hommes entre 23 et 25 ans et chez les femmes entre 18 et 20 ans. Il identifie, ensuite, des pathologies spécifiques, qui peuvent se manifester dans l'enfance (les plus graves et les plus répandues étant le paradoxisme infantile, c'est-àdire le développement non contrôlé de l'instinct sexuel, dont il sera traité plus loin, et la masturbation) et des comportements considérés comme déviants au cours de l'adolescence (homosexualité, alcoolisme et prostitution). Cette dernière période telle qu'il l'aborde a des limites incertaines et une étendue considérable, puisqu'elle peut inclure toutes les années qui précèdent la pleine maturité sexuelle.

Cet article se propose d'analyser les explications fournies par Forel quant à l'origine des perversions, qui reste attachée selon lui à une théorie de la dégénérescence, considérant que l'hérédité l'emporte toujours sur l'environnement social. Puis nous étudierons les caractéristiques des différentes formes pathologiques, illustrées par quelques cas, et les modalités élaborées par Forel pour en empêcher la propagation, à savoir des propositions eugénistes associées à des réformes éducatives et pédagogiques. L'intention est de montrer comment la réflexion de Forel se situe dans un moment de passage marqué, d'un côté, par les tout derniers développements de la scientia sexualis, née au milieu du XIX ${ }^{\mathrm{e}}$ siècle, avec pour objectif l'identification et la classification des perversions sexuelles dans l'optique de leur confinement; d'un autre côté, par la validation du processus de sexualisation de l'enfant, qui trouve une systématisation et une orientation d'ordre psychologique avec l'œuvre de Freud $^{1}$. Nous nous référons ici en particulier à la thèse développée par Arnold I. Davidson $^{2}$, lequel, reprenant l'enseignement de Michel Foucault, distingue trois phases dans la compréhension de la perversion. La première phase correspond à l'idée que la perversion est liée à une anomalie des organes reproducteurs et génitaux; dans la deuxième phase, quand bien même on a introduit la notion d'instinct, on pense encore que les anomalies sexuelles ont un fond organique dont l'origine doit être recherchée dorénavant dans les pathologies qui touchent le cerveau. Si les deux premières phases adoptent encore un mode de raisonnement (le style of reasoning dont parle Ian Hacking ${ }^{3}$ ) de type 
anatomopathologique, qui entend démontrer l'existence de telles altérations au moyen de l'analyse clinique des signes présents à l'intérieur des cadavres, en particulier dans les organes et dans les tissus ${ }^{4}$, la troisième marque l'avènement d'une interprétation proprement psychiatrique, selon laquelle les perversions sexuelles constituent des déviations fonctionnelles de l'instinct sexuel (dont les manifestations prennent le nom, souvent interchangeable, de pulsions, attitudes, satisfactions ou simplement traits psychiques) et n'ont plus aucun lien direct ou indirect avec les organes. Le grand avantage de l'affirmation selon laquelle il existe des fonctions sans liens avec un quelconque organe consiste dans le fait que des conditions apparemment éloignées, de l'hermaphrodisme à l'homosexualité, du sadisme au fétichisme, certaines étant élaborées dans le domaine du droit, d'autres dans le champ médical, peuvent être désormais répertoriées sous une même étiquette, celle de "perversion ». Davidson précise que ces trois moments ne s'enchaînent pas de façon bien définie au niveau chronologique mais qu'ils peuvent se superposer soit à une même période soit dans les travaux d'un même auteur. En fixant son attention sur la labilité et la porosité des limites entre les styles de raisonnement, on obtient une grille de lecture qui, à notre sens, peut se révéler particulièrement utile si on l'applique au cas de Forel, qui se tient en équilibre entre les deux derniers moments : enclin à une explication psychologique, il est en même temps peu disposé à abandonner l'idée d'un substrat organique, fût-il cérébral, des troubles comportementaux. Autrement efficace nous semble le lien qu'établit Davidson entre " perversion " et "processus d'individuation " : l'« invention » de la perversion a ainsi marqué le déclin de l'anatomie pathologique et la création par la pyschiatrie de types humains spécifiques comme l'hermaphrodite, l'homosexuel, le sadique et le fétichiste. De cette façon, la sexualité devient la caractéristique la plus importante de la personnalité et de l'identité humaine, ce qui représente un changement radical dans la conceptualisation et la compréhension de celle-ci. Et pas seulement : l'étude de la sexualité vise souvent à l'adoption de mesures pour lutter contre les perversions et, plus généralement, au contrôle des corps et des instincts. Et c'est ce qui se passe avec Forel, pour qui il n'y a pas d'étude de la sexualité sans recours à l'eugénisme, parfois dans ses formes les plus radicales, comme la stérilisation et l'euthanasie des nouveau-nés affectés de tares physiques et psychiques héréditaires ${ }^{5}$. Si, avec l'attention portée aux perversions naissent de nouveaux genres humains, en particulier les pervers, avec l'avènement
4. II s'agit du regard médical dont parle Foucault dans Naissance de la clinique, c'est-à-dire d'un regard plurisensoriel, qui implique non seulement la vue mais aussi le toucher et l'ouïe.

5. Sur la stérilisation comme forme radicale de ce qui a été défini comme le paradigme immunitaire cf. Esposito Roberto, Bíos. Biopolitica e filosofia, Torino, Einaudi, 2004, p. 141-142. 
6. Cf. BABINI Valeria Paola,

"Maria Montessori e Gina Lombroso. Due risposte femminili al problema della degenerazione " [Maria Montessori et Gina Lombroso. Deux réponses féminines au problème de la dégénérescence]; SIMILI Raffaella (a cura di), Scienza a due voci [Science à deux voix], Firenze, Olschki, 2006, p. 183-215. De la même auteure cf. la biographie intellectuelle de Montessori écrite en collaboration avec Luisa Lama: Una «donna nuova». II femminismo scientifico di Maria Montessori [Une « femme nouvelle». Le féminisme scientifique de Maria Montessori] Milano, Franco Angeli, 2000, 320 p.

7. Le néologisme " eugénique " a été introduit par Francis Galton, cousin de Darwin, dans Inquiries into Human Faculty and Its Development [Enquêtes sur les facultés humaines et leur développement], (London, Macmillan, 1883, p. 24-25, note 1), pour dénommer la science de l'amélioration de

la race (stock), qui devait tendre non seulement à favoriser des accouplements pertinents entre personnes de qualité physique et morale élevée, mais aussi à encourager l'étude des moyens par lesquels les races les plus aptes (more suitable) pourraient rapidement prévaloir sur celles qui l'étaient moins (less suitable). Le projet eugénique de Galton était animé d'un fort élan utopique, comme le montre sa dernière œuvre, Kantsaywhere, un récit dans lequel on imagine que tous les aspects de la vie humaine peuvent être contrôlés en vue de la sélection artificielle des individus physiquement et psychologiquement meilleurs. En outre, l'eugénisme était assez souvent comparé

par Galton à une nouvelle forme de religion, avec pour visée de guider et orienter rationellement les processus sélectifs de la nature. Sur l'utopie galtonienne cf. La Vergata Antonello, Eugénisme et utopie, Fortunati Vita, Trousson Raymond (dir.), Histoire transnationale de l'utopie littéraire et de l'utopisme, Paris, Honoré Champion, 2008, p. 761-784. de l'eugénisme on en vient à circonscrire de nouvelles catégories de sous-humains, dont les vies représentent un poids économique et sanitaire pour la société, et qui sont donc jugées indignes d'être vécues, pour reprendre le titre d'un célèbre livre publié en 1920 par le juriste Karl Binding et le psychiatre Alfred Hoche, Die Freigabe der Vernichtung lebensunwerten Lebens [Le droit de détruire la vie dénuée de valeur].

L'idée de Forel d'analyser d'un point de vue évolutionniste le développement de la vie sexuelle des hommes et des femmes, en privilégiant les aspects dysfonctionnels et dégénératifs, met au centre de son attention enfants et adolescents parce que c'est en eux que germent les pathologies qui vont ensuite se développer de façon plus complète à l'âge adulte. L'enfant est presque assimilé à un nid potentiel de perversions, selon une image répandue à l'époque - on pense ici à l'idée freudienne de l'enfant comme "pervers polymorphe ", ou encore aux travaux, en Italie, de Maria Montessori et de la fille de Cesare Lombroso, Gina Lombroso ${ }^{6}$. En ce sens, la connaissance de la sexualité juvénile n'est pas pour Forel une fin en soi, c'est-à-dire que ce n'est pas une étude exclusivement érudite ou spécialisée à mener seulement dans l'espace clos des cliniques et des asiles, mais elle constitue la base nécessaire à cette mission de réforme sociale et politique dont est investi le médecin-eugéniste. Il s'agit d'un engagement civique et d'un quasiapostolat aux accents parfois utopistes, semblables à ceux qui caractérisent l'eugénisme galtonien ${ }^{7}$ dont Forel se considère comme un précurseur. Cette préoccupation pour l'enfance anormale et malade vient ainsi de la conviction que les enfants 
constituent le cœur et la raison d'être de la famille monogamique; le défaut de traitement de la dégénérescence ébranle par conséquent les fondements mêmes du corps social et représente un risque pour sa santé physique et psychique. D'une part, les enfants doivent être soustraits aux interventions indues de leurs parents, coupables, surtout quand ils appartiennent aux classes populaires, afin de les éloigner des risques que représentent la mendicité, la prostitution, l'alcoolisme et la criminalité plus généralement. D’autre part, leurs comportements sociaux doivent faire l'objet de rigoureuses formes de contrôle visant à limiter le « danger des perversions sexuelles " et à éviter que de telles conduites ne se propagent, contaminant ainsi leurs pairs. Et c'est précisément la tendance à l'imitation de comportements moralement blâmables qui suscite chez Forel les plus grandes anxiétés, parce qu'elle peut accroître de façon artificielle l'appétit sexuel et l'orienter vers des actes considérés comme contre-nature. Quand cela advient, comme c'est le cas avec les criminels (n'oublions pas que Forel était lecteur et adepte de l'œuvre de Lombroso), l'homme retrouve des traits de son passé sauvage et féroce, mettant au défi par ses comportements " anachroniques » et impitoyables les conventions de la morale bourgeoise, et compromettant l'existence même des classes moyennes ${ }^{8}$. Le risque est encore plus grand pour les enfants, dans la mesure où le réajustement de la loi biogénétique fondamentale formulée par Ernst Hæckel permet de les considérer comme plus proches sur l'échelle évolutive des hommes primitifs et des bêtes que ne le sont les adultes ${ }^{9}$. Le travail, allié à une occupation intellectuelle, à développer dans des structures
8. Sur ce danger, même dans un contexte d'exaltation de la primauté nationale qui, certes, ne concerne pas Forel, ardent défenseur de l'internationalisme socialiste, cf. l'étude désormais classique de Mosse George L., Nationalism and Sexuality: Respectability and Abnormal Sexuality in Modern Europe [Nationalisme et sexualité respectabilité et sexualité anormale dans l'Europe moderne], New York, Howard Fertig, 1985, 232 p.

9. Selon cette loi, présentée par Hæckel en 1866 dans le second volume de sa Generelle Morphologie [Morphologie générale], chaque individu traverse au cours de son développement une succession de phases typiques du lent développement des formes ancestrales adultes, c'està-dire que l'ontogenèse est la reproduction sous une forme synthétique et accélérée de la phylogenèse. Sur le destin de la loi haeckelienne et sur son influence dans des champs du savoir parfois éloignés entre eux, cf. deux ouvrages fondamentaux : GoulD Stephen Jay, Ontogeny and Phylogeny [Ontogénèse et phylogénèse], Cambridge (Mass.), Belknap Press of Harvard University Press, 1977, 501 p. ; Rossi Paolo, Bambini, sogni, furori. Tre lezioni di storia delle idee [Enfants, rêves, fureurs. Trois leçons d'histoire des idées], Milano, Feltrinelli, 2001, $167 \mathrm{p}$. 
10. Le plus important document sur la vie de Fore reste son autobiographie publiée de façon posthume (Rückblick auf mein Leben, Zürich, Büchergilde Gutenberg, 1935) et traduite en français en 1941 (Mémoires, Neuchâtel. Baconnière).

11. DARWIN Leonard (ed.), The Life and Letters of Charles Darwin. Vol. III [La vie et les lettres de Charles Darwin], London, Murray, $1887^{2}$, p. 191-193.

12. Forel Auguste, Beiträge zur Kenntniss des Thalamus opticus und der inn umgebenden Gebilde bei den Säugethieren (Dissertation, Wien 1872) ; «Untersuchungen über die Haubenregion und ihre oberen Verknüpfungen im Gehirne des Menschen und einiger Säugethiere, mit Beitragen zu den Methoden der Gehirnuntersuchung » (Habilitation), Archiv für Psychiatrie und Nervenkrankheiten, $\mathrm{n}^{\circ} 7$ 1877, p. 393-495 spécifiques sous la surveillance d'enseignants et de médecins, sont les meilleurs moyens de défendre le processus de civilisation des êtres humains et d'éviter la rechute dans la barbarie et l'animalité. Sans jeunes gens sexuellement disciplinés, il est impossible d'espérer parvenir à une société d'adultes sains, vigoureux et moralement responsables.

\section{FOREL : PRATICIEN ET THÉORICIEN DES PERVERSIONS SEXUELLES}

Mais avant d'aller plus loin, il convient de revenir succintement sur la vie de Forel. Forel naît en 1848 à Morges, dans le canton de Vaud, d'une famille de la moyenne bourgeoisie protestante. Sa formation universitaire se déroule sous le signe de deux intérêts qui caractérisent sa future carrière de savant ${ }^{10}$ : d'une part, les recherches entomologiques, avec une attention particulière portée à la vie sociale des fourmis, qui l'amènent à voyager en Amérique du Sud et en Afrique du Nord et qui lui valent les éloges de Charles Darwin ${ }^{11}$, faisant de lui un des plus influents myrmécologues du monde. D’autre part, les études de médecine et de psychologie comparée qu'il entreprend à l'université de Zurich et qu'il perfectionne avec Theodor Meynert, à la clinique Lazarettgasse de Vienne, puis avec Bernhard von Gudden, à la KreisIrrenanstalt de Munich ${ }^{12}$. À partir d'août 1879, à l'âge de 31 ans, Forel devient directeur de la clinique psychiatrique Burghölzli de Zurich (occupant parallèlement la chaire de psychiatrie à l'université locale) jusqu'en 1898. Durant ces vingt années, il dote la clinique d'une organisation et d'une structure scientifique moderne, se battant 
pour le no-restraint, c'est-à-dire pour l'abolition des moyens de coercition utilisés dans les asiles pour le traitement des aliénés, et soulignant la nécessité d'une formation spécialisée pour le personnel. Il complète, en outre, l'étude de l'anatomie cérébrale par la pratique de l'hypnotisme à des fins thérapeutiques. Cette expérience l'amène à publier en 1889 son livre Der Hypnotismus [L'hypnotisme], qui lui vaut une enthousiaste recension de la part de Freud ${ }^{13}$. À la fin des années 1880, il devient aussi l'un des principaux animateurs du mouvement d'abstinence de l'alcool, combinant intérêt théorique et engagement social. Après avoir quitté le Burghölzli, il revient à Morges et se consacre à la publication de son livre Die sexuelle Frage [ La question sexuelle], qui paraît chez l'éditeur Ernst Reinhardt en mars 1905, la même année que Drei Abhandlungen zur Sexualtheorie [Trois essais sur la théorie sexuelle] de Freud. Le livre, dédié à sa femme Emma Steinheil, est très bien accueilli en Europe et aux États-Unis grâce aux traductions en français, italien et anglais ${ }^{14}$. Cette publication le consacre comme l'un des plus grands sexologues de son temps. Il s'agit de la première étude systématique et extensive que Forel consacre à la sexologie. En effet, jusqu'alors il s'était limité à de brèves contributions pour des revues spécialisées, concernant principalement le problème du traitement de la prostitution et les effets de l'alcoolisme sur les perversions sexuelles. L'intention du livre est de présenter à un public cultivé (tout comme KrafftEbing s'adresse aux médecins et aux juristes avec sa Psychopatia sexualis, dont la première édition remonte à $1886^{15}$ ) le problème de la sexualité humaine d'un point de vue biologique, ethnolo-
13. Forel Auguste,

Der Hypnotismus.

Seine Bedeutung und seine Handhambung

[L'hypnotisme. Sa signification et son traitement], Stuttgart, Enke, 1889. Dans les années qui suivirent, le volume fut considérablement augmenté, au point de passer des quelque 90 pages de la première édition à plus de 350 dans la cinquième édition de 1911, qui parut sous le titre : Der Hypnotismus oder die Suggestion und die Psychotherapie. Ihre psychologische, psychophysiologische und

medizinische Bedeutung [L'hypnotisme ou suggestion et la psychothérapie. Sa signification psychologique, psychophysiologique et médicale]. Pour la recension, cf. Freud Sigmund, Wiener medizinische Wochenschrift, 39, 28, 1889 , p. 1097-1100; 39, 47, 1889, p. $1892-1896$.

14. Forel Auguste, Die sexuelle Frage. Eine naturwissenschaftliche, psychologische, hygienische und soziologische Studie für Gebildete, München, Reinhardt, 1905 (trad. fr. La question sexuelle exposée aux adultes cultivés, Paris, Steinheil, 1906, d'où seront tirées par la suite les citations; trad. it. La questione sessuale esposta alle persone colte, Torino, F.lli Bocca, 1907; trad. angl. The Sexual Question. "A Scientific, Psychological, Hygienic and Sociological Study for the Cultured Classes", London, Rabman, 1908). En 1928, on compte de nombreuses traductions dont celles en suédois, en danois, en finlandais, en espagnol, en hollandais, en russe, en polonais en bulgare, en serbe, en tchèque, en portugais et en hongrois.

15. Krafft-Ebing Richard von, Psychopathia sexualis. Eine klinisch-forensische Studie [Psychopathia sexualis. Une étude médicolégale], Stuttgart, Enke, 1886. La première traduction française remonte à 1895 . 
16. Preiswerk Frank «Auguste Forel (1848-

1931) : un projet de régénération sociale, morale et raciale ", Les Annuelles, n’ 2, 1991, p. 25-50; HeLLER Geneviève, JeAnmonod Gilles,

Gasser Jacques, Rejetées, rebelles, mal adaptées. Débats sur l'eugénisme. Pratiques de la stérilisation non volontaire en Suisse romande au $x x^{e}$ siècle, Genève, Georg, 2002, 480 p. ; Gerodettı Natalia, Modernising Sexualities: Towards a Socio-Historical Understanding of Sexualities in the Swiss Nation

[Moderniser les sexualités : vers une compréhension socio-historique des sexualités dans la nation suisse], Bern, Peter Lang, 2005, 286 p. ; LeIst Anton (hrsg.), Auguste Forel - Eugenik und Erinnerungskultur [Auguste Forel - Eugénique et culture de la mémoire], Zürich, vdf, 2006, 127 p. ; KüCHENHOFF Bernhard, "The Psychiatrist Auguste Forel and His Attitude to Eugenics", History of Psychiatry, n 19 , 2008, p. 215-223; WECKER Regina, BRAunschWEIG Sabine, IMBODEN Gabriela, RItTER Hans Jakob,

Eugenik und Sexualität. Die Regulierung reproduktiven Verhaltens in der Schweiz [Eugénique et sexualité. Attitude à l'égard de la régulation de la reproduction en Suisse], 1900-1960, Zürich, Chronos, 2013, $201 \mathrm{p}$. gique, psychopathologique, hygiénique et sociologique. L'exposé théorique qui se réclame, outre que de Krafft-Ebing pour la partie psychopathologique, explicitement d'Edvard Westermark et de son History of Human Marriage [Histoire du mariage humain] (1891) pour la partie anthropologique et des réflexions d'August Bebel pour les thèmes sociaux et pour le rôle de la femme dans la société in Die Frau und der Sozialismus [La femme et le socialisme] (1879) - est illustré par de nombreux cas cliniques, examinés directement par Forel dans le cadre de sa clinique zurichoise et lors des consultations privées qu'il tint à Morges, ou bien encore glanés dans les actualités journalistiques ou les procès judiciaires. Les nombreuses éditions de La question sexuelle qui se succèdent du temps de Forel ou après sa mort laissèrent quasi inchangé le contenu de l'œuvre, dont ses contemporains appréciaient surtout le vaste programme de réformes d'inspiration socialiste qui y était présenté et qui comprenait entre autres la reconnaissance des droits des femmes, la libération du mariage des liens religieux, la licéité du divorce, l'égalité entre enfants légitimes et enfants naturels, l'introduction à l'école de l'éducation sexuelle pour garçons et filles et l'abrogation de toutes les mesures législatives et des peines infamantes édictées contre les actes sexuels qui ne causaient pas de tort au reste de la population. En même temps, Forel préconise un processus radical de sélection artificielle, invoquant les instruments coercitifs déjà rappelés qui, sous peu, allaient devenir typiques des traditions biopolitiques étatsunienne, allemande et scandinave, comme la stérilisation forcée, qu’il pratique lui-même au Burghölzli. Ses idées d'avant-garde finiront par inspirer la législation adoptée par le canton de Vaud en 1928 qui prévoyait la possibilité, pour un Conseil de santé spécifique, de recourir à la stérilisation des patients atteints d'infirmités mentales ou s'adonnant à la consommation d'alcool ou de drogues ${ }^{16}$.

Après avoir été victime en 1912 d'une hémorragie cérébrale qui lui paralyse la partie droite du visage, durant la Grande Guerre il soutient la suppression des armées et la fondation d'une "fédération supernationale des peuples ". Dans les dernières années de sa vie, il se consacre à une monumentale œuvre de myrmécologie en cinq volumes, Le monde social des fourmis du globe comparé à celui de l'homme (1921-1923), et il est parmi les fondateurs, en 1928, aux côtés de Magnus Hirschfeld et Havelock Ellis, de la Ligue mondiale pour la réforme sexuelle. Il meurt à Morges en 1931. 


\section{LE DÉVELOPPEMENT DE LA SEXUALITÉ}

\section{La théorie des instincts}

Pour comprendre la conception de la sexualité infantile et plus généralement humaine qui est celle de Forel, il faut se référer aux deux présupposés sur lesquels se fonde sa psychologie ${ }^{17}$. En premier lieu, s'appuyant sur un monisme rigoureux, Forel soutient que l'esprit (Seele), terme par lequel il désigne l'ensemble des fonctions mentales, et le cerveau (Gehin) sont une seule et même chose. Investigation psychologique et investigation physiologique coïncident parce qu'elles affrontent la même matière considérée de deux points de vue différents. L’objectif polémique est l'hypothèse dualiste, qui repose selon Forel sur « des croyances mystiques sans aucun fondement scientifique $^{18}$ " en contradiction avec les expériences scientifiques et les progrès médicaux. Le second présupposé consiste dans l'usage très large qu’il fait de la théorie héréditaire proposée par le zoologue allemand Richard Semon dans Die Mneme als erhaltendes Prinzip im Wechsel des Organischen Geschehens (1904) [La mnème comme principe conservateur dans les changements survenant dans l'organisme $\left.{ }^{19}\right]$. Ce qui attire l'attention de Forel, c'est la possibilité d'appliquer les explications de Semon, issues du champ zoologique et biologique, à l'homme et de les utiliser comme point de départ pour élaborer une théorie des instincts sur des bases physiologiques, dans laquelle l'instinct premier et fondamental est l'instinct sexuel. Du point de vue lexical, dans les versions allemandes de ses œuvres, y compris Die sexuelle Frage, Forel utilise Trieb et Instinkt comme synomymes pour indiquer l'instinct sexuel purement animal.

17. Forel Auguste, Hygiene der Nerven und des Geistes im gesunden und kranken Zustande, Stuttgart, Moritz, 1903 (trad. fr. L'âme et le système nerveux. Hygiène et pathologie, Paris, Steinheil, 1906 ; trad. angl. Hygiene of Nerves and Mind in Health and Disease, London, Murray, 1907).

18. Ibid., p. 2.

19. «Depuis Darwin, personne n'avait mieux que Richard Semon étudié les bases de la théorie de l'évolution, ni apporté autant de documents et de confirmations. Personne non plus n'avait mieux compris tout ce que la psychologie pouvait gagner à ces conceptions " (ForeL, Mémoires..., op. cit., p. 297). Cf. Schacter Daniel L., Forgotten Ideas, Neglected Pioneers: Richard Semon and the Story of Memory [Idées oubliées, Pionniers négligés : Richard Semon et l'histoire de la mémoire], Philadelphia, Psychology Press, 2001, 393 p., en particulier p. 57-76. 
20. Forel, La question..., op. cit., p. 56.

21. Au nom de son réductionisme, avec le temps Forel abandonnera presque complètement l'expression libido sexualis au profit de la théorie des instincts, en venant même à reprocher à Freud d'être tombé dans des "exagérations qui gâtent son œuvre », comme il l'affirme dans la cinquième édition de La question sexuelle (Paris, Masson, 1922, p. 618).

22. Forel Auguste, Der Hypnotismus und die Suggestive Psychotherapie [L'hypnotisme et la psychothérapie de la suggestion], Stuttgart, Enke, 19024 , p. 20.

23. Même s'il définit avec une certaine fréquence les Européens comme
« race arienne » et « race blanche », Forel ne partage pas la conception anthroposociologique de l'eugéniste français Georges Vacher de Lapouge, selon lequel les phénomènes sociaux doivent être expliqués à partir de l'analyse non pas tant des conditions matérielles que des raisons ethniques. En particulier, Forel critique la valeur de l'indice céphalique - la mesure correspondant au rapport entre la largeur et la longueur maximales du crâne, introduite vers 1840

par l'anatomiste suédois Adolf Anders Retzius

- largement utilisé par

l'anthropologie physique européenne et par le même Lapouge pour l'identification des races humaines.

24. Forel, L'âme..., op. cit., p. 34.
Moins fréquente est l'expression libido sexualis, qui pour Forel correspond au "désir (Begriede) passionné et purement sexuel des deux sexes l'un pour l'autre ${ }^{20}$ ", c'est-à-dire la façon dont l'instinct sexuel se manifeste chez l'homme. À la différence de la sexualité animale, la libido humaine excède le but de l'autoconservation et c'est cet écart qui doit être opportunément régulé et discipliné ${ }^{21}$. Se réclamant de Darwin, Forel définit l'instinct comme un "produit automatique, une intelligence cristallisée, fixée " grâce au travail plastique des nerfs ${ }^{22}$. Plus un organisme est évolué, plus grand est le nombre des instincts qui, à travers des engrammes spécifiques, sont devenus des automatismes. Forel précise que les instincts chez l'homme peuvent être naturels et artificiels. Les premiers sont des instincts ataviques hérités de nos ancêtres animaux et liés à la conservation de l'individu et de l'espèce (terme souvent confondu avec "race" par le même Forel ${ }^{23}$ ). Il s'agit de "sensations viscérales imprécises ${ }^{24}$ " ou indifférenciées, c'est-à-dire non localisables, comme la faim, la soif et l'instinct sexuel, qui peuvent parfois dégénérer en prenant des formes incontrôlées ou pathologiques. Les instincts artificiels, au contraire, sont des « passions » qui se développent à travers l'exemple et l'habitude, comme la gourmandise, le jeu, le sport et l'alcool, et qui peuvent aboutir à des formes de dégénérescence affectant l'organisme tout entier.

Cette théorie des instincts permet à Forel d'affirmer, en premier lieu, qu'une grande part de l'activité cérébrale est subconsciente et peut être réactivée à l'aide de procédures d'analogie et d'induction. C'est ainsi qu'on autorisera le recours à l'hypnose comme technique de psychothérapie des 
perversions sexuelles. Le modèle est celui de l'autosuggestion mis au point par Bernheim dans De la suggestion et ses applications à la thérapeutique (1886), qui rend possible, selon Forel, la réactivation et le déchiffrement des engrammes latents $s^{25}$. En second lieu, la théorie des instincts permet d'harmoniser transmission des caractères acquis et darwinisme précisément à un moment où la théorie de Darwin est critiquée de toutes parts ou même réfutée $e^{26}$. La mnème fournit les matériaux héréditaires parmi lesquels la sélection choisit les plus avantageux pour l'adaptation, transformant les instincts en automatismes fixés génération après génération par les lois de l'hérédité : en bref, « la mnème bâtit, la sélection trie ${ }^{27}$ ». L'insistance sur la lenteur des mécanismes héréditaires n'exclut pas complètement la possibilité de mutations imprévues, comme celles dont avait parlé le botaniste hollandais Hugo De Vries, dues à la re-combinaison des patrimoines génétiques des géniteurs.

\section{Hérédité et milieu}

Pour Forel, l'action de l'hérédité est toujours plus forte que celle du milieu. Étant en grande partie déterminés par les dispositions reçues des parents, on est libres dans la mesure où l'on parvient à adapter ces caractéristiques de départ aux circonstances matérielles, intellectuelles et morales dans lesquelles se déroule sa propre vie : "l'homme le plus libre est le plus adaptable ${ }^{28}$ ". Dans le cas des maladies nerveuses et mentales et des anomalies sexuelles, les causes externes peuvent seulement activer ou atténuer des dispositions héréditaires déjà présentes. L'éducation elle-même ne peut pas créer de nouvelles attitudes mais seulement agir sur celles qui existent déjà. Cela signifie que pour Forel, quand manque la disposition, même les séductions les plus puissantes ne peuvent la faire naître. Il n'existe donc pas de "vice acquis » : les vices dépendent toujours d'une disposition héréditaire, plus ou moins pathologique, qui se développe tôt ou tard du fait de l'influence du milieu.

La connaissance des lois de l'hérédité, alliée à l'anatomie comparée et à la biologie animale, explique en quoi l'ontogénèse reproduit la phylogénèse, même si Forel ne reconnaît pas à l'intérieur du cycle du développement sexuel de l'individu des phases nettes mais seulement des périodes plutôt étendues, qui ne sont pas délimitées a priori par l'âge ni par le développement organique mais qui sont liées à l'influence souvent imprévisible de facteurs internes et externes. Au début, l'enfant présente « des mélanges confus de sensations ${ }^{29}$ ":

\author{
25. Bugmann Mirjam, \\ Hypnosepolitik. Der \\ Psychiater August Forel, das \\ Gehirn und die Gesellschaft \\ (1870-1920) [La politique \\ de l'hypnose. Le psychiatre \\ Auguste Forel, le cerveau \\ et la société], Köln-Weimar- \\ Wien, Böhlau Verlag, 2015, \\ $336 \mathrm{p}$. \\ 26. Bowler Peter J., The \\ Eclipse of Darwinism. Anti- \\ Darwinian Evolution Theories \\ in the Decades Around 1900 \\ [L'éclipse du darwinisme. \\ Les théories de l'évolution \\ anti-darwiniennes dans les \\ décades autour de 1900], \\ Baltimore (MD), The Johns \\ Hopkins University Press, \\ 1983, $291 \mathrm{p}$ \\ 27. Forel, L'âme..., op. cit., \\ p. 85. \\ 28. Forel Auguste, Mahaim \\ Albert, Crime et anomalies \\ mentales constitutionnelles, \\ Genève, Kündig, 1902, p. 7. \\ 29. Forel, L'âme..., ibid., \\ p. 110.
}


30. ForeL, L'âme..., ibid., p. 111. un enfant de 4 ans, par exemple, ne se rappelle pas les événements qui ont marqué sa première année de vie mais ceux-ci sont quoiqu'il en soit inscrits dans sa mémoire organique. Les instincts commencent à se manifester après que le système nerveux central se soit correctement formé et que ses fibres se soient différenciées. Le complet développement de toutes les fonctions nerveuses et la maturité sexuelle, c'est-à-dire l'entrée dans l'âge adulte, est atteint chez les hommes entre 23 et 25 ans, chez les femmes entre 18 et $20^{30}$. Durant l'adolescence, l'attention ne se concentre pas tant sur l'acte que sur le désir, qui se manifeste surtout dans l'activité onirique de type érotique, donnant lieu chez les garçons à des pollutions nocturnes. L'imagination peut être excitée aussi artificiellement par la lecture de récits pornographiques et par la consommation d'alcool; Forel parle à ce propos de formes de «brigandage social " parce que visant à l'exploitation économique de la sexualité par le grand capital.

Les différences dans le développement de la sexualité masculine et féminine sont expliquées en mettant en cause la constitution physiologique et le rôle actif ou passif tenu pendant l'acte. Les garçons sont attirés ou par ce qui est étrange et exceptionnel, ou par ce qui est sain et épanoui. Dans la première catégorie rentre la perception d'odeurs inhabituelles, ainsi que la vue de parties du corps généralement couvertes, d'habits et d'ornements voyants, et de femmes qui ne leur sont pas familières. Cela justifierait leur répulsion naturelle à l'égard de leurs sœurs, que leur fréquentation habituelle amène à ne pas considérer comme des objets de désir. L'attirance pour la diversité vaut aussi pour les jeunes gens appartenant aux peuples sauvages, mais avec un signe opposé : puisqu'ils sont habitués à la nudité, leur appétit est excité par la vue de filles vêtues ou qui ont recours à des embellissements particuliers. Cette attention pour l'aspect extérieur indique une forme de fétichisme qui rentre dans la normalité, puisque le désir suscité par la vue de parties du corps ou objets inanimés est tourné, en dernière instance, vers l'individu qui les donne à voir. L'instinct sexuel peut, d'autre part, être réveillé par la présence de corps épanouis, de peaux saines et d'odeurs ordinaires. Dans le cas des filles, l'appétit sexuel présente plus facilement des excès, qui vont du débridement à l'absence totale de désir. Ce n'est pas seulement la disposition anatomique des zones érogènes qui est différente par rapport à l'homme mais la motivation même qui est à la base de l'acte sexuel : plus que la volupté, les jeunes femmes cherchent l'amour, entendu surtout comme protection et stabilité. Étant elle-mêmes très liées à 
leurs propres instincts et à leurs propres habitudes, elles apprécient chez leur partenaire surtout l'audace et l'esprit d'initiative.

\section{LES PERVERSIONS DE L'ENFANCE}

\section{Le fondement biologique des dégénérescences sexuelles}

Comme on l'a vu dans l'introduction, Forel oscille constamment entre les deuxième et troisième phases de l'explication des perversions proposée par Davidson, mettant l'accent tantôt sur les aspects biologiques et neurologiques, tantôt sur les aspects psychiatriques. D'un côté, il fait un large usage du concept d'instinct sexuel et il soutient que " toute aliénation mentale repose sur un trouble des fonctions cérébrales ${ }^{31}$ ». De l'autre, il a tendance à considérer que la différence entre organique et fonctionnel est de degré et non de nature. Par "problèmes mentaux et nerveux organiques » on doit entendre, selon lui, les altérations pathologiques qui conduisent à la détérioration du système nerveux central, générant des lésions anatomiques reconnaissables. On peut, par contre, qualifier de "fonctionnels" tous les problèmes mentaux et nerveux qui ne reposent pas sur une lésion matérielle immédiatement reconnaissable mais sur une altération de l'état physico-chimique de la substance nerveuse due à l'action de microbes. Ce qui distingue un problème organique d'un problème fonctionnel, ce n'est donc pas la lésion mais sa gravité. En général, les problèmes organiques sont difficilement curables parce que les cellules cérébrales, une fois endommagées, ne parviennent pas à se régénérer, tandis que les problèmes fonctionnels étant provoqués par des agents pathogènes sont curables à l'aide d'une prophylaxie appropriée. C'est pourquoi Forel propose de remplacer « fonctionnel » par « réparable».

Partant de ces considérations, il adopte un double registre dans l'explication de la perversion (dénommée tantôt Perversion tantôt Abnormität). Il ouvre au modèle psychologique qui s'intéresse aux phases de développement psychosexuel de l'enfant, paradigme qui s'imposera seulement avec les études de Freud, mais en même temps et de façon sans doute plus déterminante, il reste fixé au concept de "dégénérescence », typique de la sexologie de la fin du XIX ${ }^{\mathrm{e}}$ siècle $^{32}$. La notion de " dégénerescence " avait été introduite dans le champ psychiatrique en 1857 par l'aliéniste Bénédict-Augustin Morel, praticien de l'hôpital de Saint-Yon, pour indiquer " une déviation maladive du type normal de l'humanité $^{33}$ " et, entre la fin du XIX ${ }^{\mathrm{e}}$ et le début du $\mathrm{Xx}^{\mathrm{e}}$ siècles, elle avait connu une
31. Forel, L'âme..., op. cit., p. 136.

32. Chaperon Sylvie, « Les fondements du savoir psychiatrique sur la sexualité déviante au XIX ${ }^{e}$ siècle », Recherches en psychanalyse, n², 2010, p. 276-285.

33. Morel BénédictAugustin, Traité des dégénérescences physiques, intellectuelles et morales de l'espèce humaine et des causes qui produisent ces variétés maladives, Paris, Baillière, 1857, p. 5 
34. Pick Daniel, Faces of Degeneration: a European Disorder, c.1848-c. 1918 [Visages de la dégénérescence : un trouble européen], Cambridge, Cambridge University Press, 1989, 275 p. Cf. aussi GILMAN Sander L., Difference and Pathology: Stereotypes of Sexuality, Race, and Madness [Différence et pathologie : les stéréotypes à propos de la sexualité, de la race et de

la folie], Ithaca-New York, Cornell University Press 1985, 292 p. (en particulier p. 191-216) ; CoffIN JeanChristophe, La transmission de la folie, 1850-1914, Paris,

L'Harmattan, 2003, 286 p.

35. Foucault Michel, Les anormaux. Cours au Collège de France, 1974-1975, Paris, Seuil/Gallimard, 1999, 351 p.

36. Crozier Ivan, « La sexologie et la définition du "normal" entre 1860 et 1900 », Cahiers du Genre, $\mathrm{n}^{\circ} 1,2003$, p. 17-37.

37. Forel Auguste, Sexuelle Ethik. Ein Vortrag gehalten am 23. Marz 1906 auf Veranlassung des Neuen Vereins in München [Éthique sexuelle. Discours prononcé le 23 mars 1906 à Munich à l'initiative de la Neuen Vereins], München, Reinhardt, 1906, p. 18.

38. Forel Auguste, Ethische und rechtliche Konflikte im Sexualleben in und ausserhalb der Ehe [Conflits éthiques et juridiques dans la vie sexuelle dans le cadre et en dehors du mariage], München, Reinhardt, 1909. diffusion extraordinaire dans des domaines très divers allant de l'anthropologie criminelle à la médecine, de la sexologie à la sociologie, jusqu’à l'esthétique, devenant une sorte d'European disorder ${ }^{34}$. Ce qui fut déterminant pour Forel, ce n'est sans doute pas tant l'œuvre de Morel, qu'il ne connaissait probablement pas de façon directe, que la médiation de Krafft-Ebing et du psychiatre allemand Wilhelm Griesinger, lesquels, dans le contexte scientifique de l'Europe centrale, se servirent de la dégénérescence pour engager un processus de « médicalisation de l'anormal ${ }^{35}$ ». Par " dégénérescence " (Entartung), Forel désigne toutes les anomalies de nature organique par rapport à une normalité définie en termes plus moraux que médicaux, comme cela arrivait souvent dans les œuvres des sexologues de son temps ${ }^{36}$. L'homme peut être dit sexuellement normal, s'il est modéré dans ses comportements et s'il est capable de contrôler ses propres instincts, les orientant dans un sens hygiénique et eugéniste. Il en résulte que l'instinct sexuel, quand il est utile à la préservation de l'espèce, est positif; autrement il est négatif et doit être discipliné ${ }^{37}$. Dans cette perspective, la majeure partie des décisions liées à la vie sexuelle de l'individu prennent la valeur de conflits de nature éthique, qui seront résolus en tenant compte de leurs possibles effets sur la collectivité tout entière ${ }^{38}$. Le recours à la dégénérescence présente au moins trois avantages pour Forel : a) il permet un solide ancrage à la transmission héréditaire des pathologies mentales et physiques, avec pour conséquence la relativisation de la différence entre anomalies sexuelles héréditaires et acquises proposée par Krafft-Ebing; b) il permet la réévaluation des signes morphologiques et organiques liés aux maladies et perversions dans une perspective anatomopathologique; c) il autorise une critique plus générale du progrès, de la civilisation et du capitalisme occidental (qualifié d' " américanisme »), critique cruciale pour la vision socialiste soutenue par Forel et certes non étrangère à de larges secteurs de l'eugénisme européen.

\section{Le paradoxisme infantile}

Parmi les dégénérescences de la sexualité infantile, Forel fait figurer le " paradoxisme ", c'est-à-dire le développement précoce de l'instinct sexuel, lequel se manifeste en général entre 7 et 8 ans mais, dans certains cas, est déjà évident à partir de 3 ou 4 ans. La caractéristique de cette déviance est qu'elle apparaît spontanément, sans qu'il soit besoin d'une quelconque excitation externe et avant, donc, que l'appareil reproducteur soit complètement formé. À ce propos, 
dans La question sexuelle Forel rapporte deux cas qu'il a analysés au Burghölzli. Le premier concerne un enfant de 7 ans, fils d'une prostituée et d' " une sorte de satyre ayant commis les excès les plus intenses ${ }^{39}$ ", capable d'attirer des fillettes de son âge ou même plus jeunes en leur offrant des bonbons. Après les avoir conduites derrière des buissons, il commet des violences sexuelles. Interné dans la clinique zurichoise, l'enfant continue à se montrer sexuellement indiscipliné et tente même d'attirer un garçon plus âgé mais pas encore adulte. Et cela en dépit du fait que ses organes sexuels soient encore ceux d'un enfant non pubère et ne montrent aucun signe d'anormalité. Parfois, les conséquences du paradoxisme peuvent se révéler fatales, comme dans le second cas cité par Forel, qui implique une fillette de 9 ans. Cette dernière a l'habitude de satisfaire les plaisirs sexuels de ses deux petits frères. Mais ses gestes trop véhéments finissent par provoquer de graves lésions à la vessie et à l'urêtre de l'un des deux et provoquent la mort de l'autre. Dans le cas du paradoxisme, les enfants jouent un double rôle dans la mesure où ils manifestent des appétits anormaux et ils sont d'un autre côté victimes d'actes de pédophilie ${ }^{40}$. Le paradoxisme peut se manifester également à un âge avancé, prenant alors le nom de " pédérose ». Un des exemples rapportés concerne un artiste qui fut attiré dès sa jeunesse par des fillettes entre 5 et 10 ans, tandis que les filles plus âgées et les femmes adultes le laissaient complètement indifférent. Pendant toute sa vie cet homme parvint à contrôler son instinct, se limitant à tenir des fillettes sur ses genoux et à les caresser, au prix toutefois d'une grave dépression mélancolique ${ }^{41}$.

\section{La masturbation}

Entre la fin du XIX ${ }^{\mathrm{e}}$ siècle et le début du $\mathrm{Xx}^{\mathrm{e}}$, on assiste à une évolution de la perception de la masturbation au sein de la sexologie. La tradition de stigmatisation du sexe en solitaire, inaugurée en 1760 par le médecin suisse SamuelAuguste Tissot dans son livre, L'onanisme ${ }^{42}$ est moins nette. Il y a, par exemple, des changements terminologiques - Ellis introduit le terme d' " auto-érotisme " en 1899 et, en 1907, le médecin français Georges Saint-Paul, parle d'" autophilie $^{43}$ ». Néanmoins la proscription pour dangerosité, tant au niveau moral que physique, de la pratique masturbatoire persiste : le même Krafft-Ebing la considère comme un élément fondamental de l'étiologie des perversions et le symptôme principal (dans certaines circonstances, même la cause) d'une grande part des comportements sexuels alors considérés comme déviants ${ }^{44}$.

\author{
39. Forel, La question..., \\ op. cit., p. 240. \\ 40. Ibid., p. 240-241. \\ 41. Ibid., p. 279. \\ 42. LAQUEUR Thomas, \\ Le sexe en solitaire. \\ Contribution à l'histoire \\ culturelle de la sexualité \\ (2003), Paris, Gallimard, \\ 2005, p. 375-398. \\ 43. Chaperon Sylvie, Les \\ origines de la sexologie \\ (1850-1900), Paris, Payot, \\ 2012, p. 128-130. \\ 44. Oosterhuis Harry, \\ Stepchildren of Nature. \\ Krafft-Ebing, Psychiatry, \\ and the Making of Sexual \\ Identity, Chicago, University \\ of Chicago Press, 2000, \\ 304 p., en particulier p. 57.
}


45. FouCAULt, Les anormaux..., op. cit., p. 217-248.

46. ForEL, La question..., op. cit., p. 255

Au sein de ce débat, la position de Forel tend vers une dévaluation des effets nocifs de l'onanisme, comme le montre sa classification de ses diverses formes. En premier lieu, il y a la "masturbation compensatrice ", due à l'impossibilité de satisfaire un besoin naturel : particulièrement répandue dans les internats scolaires, elle est peu dangereuse au niveau social mais provoque une dépression nerveuse et mentale due à l'incapacité de dominer sa propre volonté. En second lieu, il faut considérer la masturbation induite par des " irritations accidentelles ", comme la phimosis et le prurit suscités par des infections des zones génitales : « innocente » quant aux causes, cette forme d'onanisme doit être soignée, dans un cas par la circoncision et dans l'autre par l'usage d'antiseptiques. Il y a, en troisième lieu, la masturbation induite par l'exemple et par l'imitation dans des contextes scolaires ou dans la rue, qui peut rendre l'enfant impuissant, paresseux et craintif, et causer des troubles de la digestion et de la nutrition. De ces trois habitudes on guérit aisément grâce à l'exercice physique en plein air, à une surveillance plus vigilante des adultes, à l'autosuggestion et surtout à des rapports sexuels avec l'autre sexe. Par contre, on ne peut pas éradiquer la quatrième forme de masturbation, liée à l'hyperesthésie sexuelle et au paradoxisme. Forel la mentionne en quelques lignes seulement pour en souligner les aspects chroniques, pour affirmer l'impossibilité d'une guérison complète étant donné sa nature héréditaire, et pour soutenir qu'il s'agit d'un phénomène habituel surtout chez les fillettes. Si, chez les hommes, l'auto-stimulation induit un état de dépression, les femmes, elles, recherchent volontairement la « répétition " et l'« intensité " de l'expérience érotique.

Pour Forel, donc, la masturbation reste une tentative de satisfaire son propre instinct de façon anormale, ce qui s'accompagne parfois d'idiotie et de névrose. Toutefois, il adresse une critique de l'intérieur à l'image traditionnelle de l'enfant onaniste transmise par la littérature sexologique et enracinée aussi dans la représentation populaire ${ }^{45}$. Les onanistes ne sont pas du tout des « figures pâles, terrifiées et gémissantes mais bien plutôt des individus lubriques, qui se transforment volontiers en don Juan insolents ${ }^{46} »$. Cette interprétation erronée du phénomène vient de ce que l'on a confondu la cause et l'effet : ce n'est pas tant la masturbation en soi qui détermine la faiblesse organique et psychique (si l'on s'en tient aux effets, elle n'est pas très différente d'une pollution nocturne) que le manque d'auto-contrôle qui induit les enfants à contracter ce vice. Voilà alors que, répondant aux nombreuses lettres qui lui sont envoyées par des jeunes 
gens s'adonnant à la masturbation, Forel cherche à bousculer quelques lieux communs. Face au cas d'un garçon de 18 ans qui impute sa faible application dans ses études au fait d'avoir pratiqué une forme compulsive d'onanisme entre 12 et 16 ans, Forel affirme que son état mental est dû à l'hypocondrie et que pour le reste son attitude peut être considérée comme «sexuellement normale ${ }^{47}$ ". À un jeune homme qui raconte qu'il n'est pas parvenu à vaincre ni par le mariage ni par la prière la « mauvaise habitude » qu'il pratique compulsivement depuis qu'il est étudiant et qui cause, entre autres, mélancolie, inquiétude, insomnies et vertiges, Forel écrit qu’il est victime de ses "préjugés » et que les causes véritables de sa dépression nerveuse sont imputables à un mélange de " psychopathie et naïveté ${ }^{48}$ ".

\section{LES COMPORTEMENTS DÉVIANTS DANS L'ADOLESCENCE}

\section{L'homosexualité}

Une dernière forme de masturbation juvénile identifiée par Forel est l' " onanisme essentiel » pratiqué par les « invertis sexuels»-expression par laquelle il désigne les homosexuels, aussi bien masculins que féminins. Dans le débat entre les tenants de l'hérédité et ceux du milieu, Forel estime que l'inversion est une perversion sexuelle congénitale, due non pas à l'action négative d'influences externes vicieuses, mais à un « hermaphrodisme partiel »du sujet, donc à une cause biologique : tandis que les glandes et les organes génitaux présentent les caractères d'un sexe, le cerveau a les caractères de l'autre. Il s'agit d'une déviation de l'appétit sexuel, lequel naturellement devrait viser à la procréation. Dans les deux cas, l'inversion est attribuable à trois caractéristiques principales - précocité, violence et intensité de l'instinct - qui se présentent de façon encore plus exaspérée chez les "tribades ", comparées à de véritables don Juan féminins pour ce qui est de leur frénésie érotique, laquelle les pousse assez souvent à organiser des orgies en bonne et due forme : "Un orgasme succède à l'autre, nuit et jour, presque sans interruption; néanmoins ces nymphomanes inverties ne sont pas extrêmement fréquentes. Leur jalousie ne cède en rien à celle des invertis masculins ou même la surpasse ${ }^{49}$. " Grâce à leur volupté, il est donc particulièrement facile, pour ces inverties, de séduire des filles hétérosexuelles, qui par ingénuité et confiance dans l'amour cèdent à leur affection, les prenant au début pour des hommes et puis finissant par tomber amoureuses d'elles.

\author{
47. ForeL, Sexuelle Ethik..., \\ op. cit., p. 45-46. \\ 48. Ibid., p. 40-41. \\ 49. ForeL, La question..., \\ op. cit., p. 275. II est curieux \\ de constater comment, en \\ parlant de «tribades » (et \\ non pas du phénomène \\ du tribadisme), Forel ne \\ fait référence ni à Paolo \\ Mantegazza, doyen de \\ l'anthropologie physique \\ italienne, dont il cite à \\ plusieurs reprises $\mathrm{La}$ \\ fisiologia dell'amore [ $\mathrm{La}$ \\ physiologie de l'amour], de \\ 1873, ni à Lombroso, mais \\ encore une fois à Krafft- \\ Ebing et surtout au cas, \\ par lui analysé, du comte \\ Sandor.
}


50. Rosario Vernon A. (ed.), Science and Homosexualities [Science et homosexualités], New York-London, Routledge, 1997, 308 p. ; ReVEnin Régis,

"Conceptions et théories savantes de l'homosexualité masculine en France, de la monarchie de Juillet à la Première Guerre mondiale ",

Revue d'Histoire des Sciences Humaines, $n^{\circ} 2$, 2007, p. 23-45.

51. Forel Auguste, « Die Theorie Dr Ammons über die Homosexualität », Archiv für Rassen- und Gesellschaftsbiologie, $n^{\circ} 5$, 1909, p. 803-805.

52. Forel, La question..., op. cit., p. 269.

53. Ibid., p. 266.
À travers cette lecture pathologique de l'homosexualité, Forel prend ses distances avec ce qu'il estime être des tentatives de « normalisation » opérées par Karl Heinrich Ulrichs, le juriste allemand qui avait forgé le terme " urningi » pour désigner les homosexuels et en avait donné une interprétation d'ordre psychologique, et par Hirschfeld, qui avait considéré en termes physiologiques l'inversion comme une forme de sexualité intermédiaire entre le masculin et le féminin. Un véritable «troisième sexe ». Ni maladie ni type de dégénérescence, pour Hirschfeld l'homosexualité était une simple variation, comme celles que l'on rencontre fréquemment dans les règnes animal et végétal ${ }^{50}$. De son côté, Forel continue fermement d'assimiler homosexualité et dégénérescence, ce qui lui semble confirmé par le nombre élevé de psychopathes et de névrosés présents parmi les invertis. Peu convainquante lui apparaît également la thèse de l'anthropologue Otto Ammon, qui avait provoqué un débat animé sur l'Archiv für Rassen-und Gesellschaftsbiologie [Archive pour la biologie des races et de la société], et selon laquelle la masturbation était à l'origine de l'homosexualitée1. Pour Forel, au contraire, l'onanisme est simplement une pratique liée à l'inversion mais on ne peut le considérer comme en étant la cause.

Les tendances homosexuelles se manifestent au cours de l'enfance et de l'adolescence par l'adoption spontanée d'attitudes qui appartiennent au sexe opposé et qui peuvent avoir trois issues différentes. Les personnalités les plus fragiles, une fois découvertes, se laissent aller à un pessimisme exaspéré : souvent incapables de supporter la honte et la réprobation sociale, elles peuvent en arriver à se suicider. Le deuxième cas concerne les invertis qui se fient aux conseils d'amis et de médecins sans scrupules et ignorants qui leur suggèrent de contracter un mariage avec une femme. Ils commencent par fréquenter les bordels pour prouver leur virilité puis ils s'engagent à contre-cœur dans la vie conjugale. Mais il s'agit-là, selon Forel « de la plus grande absurdité et en même temps de la plus mauvaise action ${ }^{52}$ " qu'ils puissent commettre, dans la mesure où ils mortifient continuellement leurs femmes par leur infidélité. Ces unions ne peuvent aboutir qu'au divorce et ceux qui les encouragent se rendent responsables d'un crime contre la société. Le troisième et dernier cas concerne ces invertis qui, en proie au cynisme et à un libertinisme immodéré s'associent à la « confrérie secrète » des homosexuels, " une sorte de franc-maçonnerie qui se reconnait au moindre signe, qui se flaire pour ainsi dire ${ }^{53} »$. 
De même que pour l'onanisme, l'attitude de Forel à l'égard de l'homosexualité est empreinte d'une certaine prudence et orientée vers la dépénalisation et la décriminalisation. Bien qu'il parte du présupposé qu'une vie sexuelle saine devrait être dirigée vers le " mariage civil », entendu comme " un contrat entre deux personnes de sexes différents qui se proposent pour but commun la reproduction de l'espèce humaine ${ }^{54}$ ", il estime que l'amour homosexuel est « assez innocent » et même moins dangereux au niveau social que la prostitution, parce qu'il ne donne pas lieu à une descendance et est destiné à s'éteindre graduellement par l'action de la sélection naturelle. La loi devrait interdire les mariages entre un inverti et une femme mais non les rapports sexuels entre hommes consentants, qui sont insignifiants du point de vue éthique (juridique et politique) dans la mesure où ils ne nuisent pas à la santé de l'espèce. C'est pourquoi Forel réclame, tout comme Krafft-Ebing et la majeure partie des sexologues de l'époque, l'abrogation du tristement célèbre paragraphe 175 du Code pénal allemand, qui condamne les rapports entre des personnes de même sexe à quatre années de prison ${ }^{55}$. Les seules circonstances où l'amour homosexuel doit être interdit sont celles où il s'adresse à des aliénés ou à des mineurs, lesquels doivent être protégés contre toutes formes d'abus. Écoles, églises et hôpitaux psychiatriques sont les lieux où se cachent les dangers les plus redoutables, puisque les adultes peuvent facilement profiter de leur rôle et de leur autorité sans trop de risques.

\section{L'alcoolisme}

Toutes les prédispositions héréditaires - y compris la prostitution, la plus grave perversion qui accable l'adolescence avec l'homosexualité - ont besoin d'une cause occasionnelle déclenchante pour pouvoir se manifester. Et cette cause, dans la majeure partie des cas, est l'alcool. D'un côté, Forel admet le lien entre dégénérescence sexuelle et alcool tel que codifié par Morel qui, dans son Traité des dégénérescences, avait énoncé une " loi de dégradation progressive " selon laquelle, dans l'espace de quatre générations, moyennant une aggravation des symptômes, on aboutissait à la stérilité de la famille de l'alcoolique. D'un autre côté, il suit un schéma typique de la propagande des sociétés anti-alcooliques suisses et allemandes de la fin du XIx ${ }^{e}$ siècle. Partant d'une critique des " préjugés » qui circulent sur les bienfaits de l'alcool, on dénonce ses dommages pour l'individu et la société et on finit par proposer des outils de lutte contre sa

\author{
54. ForeL, La question..., \\ op. cit., p. 409. \\ 55. Delessert Thierry, « Entre \\ justice et psychiatrie : \\ l'homosexualité dans le \\ projet de Code pénal suisse \\ (1918) ", Gesnerus. Swiss \\ Journal of the History of \\ Medicine and Sciences, \\ n० 62,2005 , p. 237-256.
}


56. Philippoz Chantal Ostorero, « Alcoolisme et dégénérescence. Le discours antialcoolique des élites vaudoises au tournant du siècle », Equinoze, n 6 , 1991, p. 143-159. Cf. aussi Germann Urs, «Alkoholfrage und Eugenik: Auguste Forel und der eugenische Diskurs in der Schweiz », Traverse.

Zeitschrift für Geschichte, $n^{\circ}$ 1, 1997, p. 144-154; Grosse Judith, SPÖRING Francesco, TSCHURENEV Jana (hrsg.), Biopolitik und Sittlichkeitsreform. Kampagnen gegen Alkohol, Drogen und Prostitution 1880-1950 [Biopolitique et réforme de la moralité. Campagnes contre l'alcoolisme, la drogue et la prostitution], Frankfurt-New York, Campus, 2014, 385 p.

57. Forel, La boisson dans les mœurs, 1893, p. 5-6.

58. Forel, La question..., op. cit., p. 36. consommation ${ }^{56}$. Au-delà de cette toile de fond, les particularités de la réflexion de Forel se trouvent dans le lien, établi de la façon la plus stricte par rapport au passé, entre alcoolisme, sexualité et transmission héréditaire, et dans l'attention constante qu'il porte non seulement à la santé du prolétariat urbain mais aussi à celle des enfants et des jeunes, les plus exposés selon lui à la dépendance aux narcotiques (outre l'alcool : tabac, hashish, éther et morphine).

Dans ses nombreuses interventions, Forel soutient avant tout l'idée que l'alcool n'a aucun avantage nutritionnel pour l'homme et que sa consommation ne renforce pas plus la puissance physique qu'elle ne stimule l'activité mentale. Il s'agit seulement de "préjudices ", alimentés au niveau populaire par les sociétés de production intéressées à la vente des boissons, et par les décisions de gouvernements qui se laissent facilement corrompre par l'argent de la grande industrie. Au contraire, l'alcool est un poison qui provoque l'intoxication du cerveau et la détérioration de nombreux organes internes comme le cœur, le foie et les reins. Se référant aux recherches d'Emil Kræpelin et du medecin suisse Gustav von Bunge, Forel affirme en outre qu'il n'y a aucune différence entre sa consommation modérée et l'alcoolisme chronique. Même la prise de doses légères peut avoir des effets désastreux sur la santé physique et psychique de l'individu : en user ou en abuser reviennent au même. Exemplaire à cet égard est une expérience qu'il a lui-même menée sur des élèves dont l'âge allait de 10 à 15 ans $^{57}$. Pendant plusieurs mois il leur a été administré, au déjeuner et au dîner, une dose limitée de vin consistant en un tiers de verre pour les plus jeunes et un demi-verre pour les plus âgés. Les élèves se montraient moins concentrés pendant les cours, plus enclins au sommeil et moins capables de prendre des décisions. Cette paralysie des freins psychiques (raison, sentiment et volonté), au lieu d'inhiber ou d'affaiblir les instincts sexuels, déterminait le reveil impétueux de la passion, qui augmentait d'intensité tant qu'elle n'était pas satisfaite.

Forel souligne les retombées des effets négatifs de l'alcool sur la collectivité tout entière : la détérioration du plasma germinal peut se transmettre par voie héréditaire par le biais du phénomène dénommé « blastoftorie ", lequel consiste dans la perturbation pathologique de la mnème individuelle avec pour conséquence l'apparition de tares et de dégénérescences. Les enfants des alcooliques ont une haute probabilité de devenir « soit idiots, soit épileptiques, soit déséquilibrés au point de vue mental ${ }^{58}$ " ou de mourir jeunes à cause de leur faiblesse constitutionnelle. 


\section{La prostitution}

Les plus grands risques de l'alcool pour la sexualité humaine se concentrent entre les âges de 10 et 17 ans, quand les garçons, sous l'effet de l'ivresse alcoolique s'adonnent à des rapports occasionnels, contractant des maladies vénériennes (syphilis et gonorrhée) et que les filles de familles pauvres commencent à se prostituer. L'alcool rend possibles des comportements brutaux et agit comme moyen de raccolage envers des psychologies déviées, comme celles des prostituées, "individus pathologiques ", c'est-à-dire hystériques, nymphomanes et psychopathes, "de naissance amorales, stupides, paresseuses, menteuses ${ }^{59}$ " et au caractère suggestionnable, ce qui en fait la proie des proxénètes, qualifiés par Forel d'individus oisifs, vagabonds et dépravés, qui se font entretenir par les femmes.

Le phénomène, qui a un lien étroit avec la vie malsaine menée dans les grandes villes, implique presque toutes les classes sociales. En sont victimes, d'une part, les jeunes filles appartenant aux familles ouvrières qui, du fait de leur extrême misère, vivent dans la promiscuité et le manque d'hygiène; d'autre part, des adolescentes provenant de la petite bourgeoisie, couturières et domestiques, contraintes de se prostituer pour arrondir leurs maigres salaires : «Les toutes jeunes filles, encore presque des enfants, sont, ne l'oublions pas, à la fois les plus faciles à séduire et les plus recherchées ${ }^{60}$. " Il y a, enfin, des femmes riches qui pratiquent le métier par défaut de sens moral et par amour de l'art. Le long chapitre sur la prostitution dans La question sexuelle est exclusivement réservé aux femmes (plus facilement esclaves de leurs habitudes, comme on l'a $\mathrm{vu})$, tandis que la prostitution masculine est traitée en peu de lignes et associée à l'inversion sexuelle et à la pédophilie. Forel se limite à parler de l'existence " en certaines villes de bordels d'hommes, dans lesquels de jeunes garçons se livrent pour de l'argent à la pédérastie » et d'" enfants [qui] doivent enfin être tenus en réserve pour certains roués, raffinés et riches, ou pour ceux qui sont atteints de pédérose ${ }^{61} "$.

La solution contre le proxénétisme ne consiste pas dans sa réglementation, considérée comme une mesure «hypocrite " : la tolérance des bordels suppose que les autorités gouvernementales, les forces de l'ordre et les médecins se rendent complices de la corruption et de la dépravation morale de la jeunesse. Pour Forel, qui suit en cela la proposition de la féministe anglaise Josephine Butler, l'abolition complète du phénomène est souhaitable. Cela permettrait

\author{
59. FOREL, La question..., \\ op. cit., p. 343. \\ 60. Ibid., p. 336 \\ 61. Ibid., p. 346
}


62. Forel Auguste, « Einige Worte über die reglementierte Prostitution in Kiew und über die sexuelle Hygiene », CorrespondenzBlatt für Schweizer Ärzte, no 19, 1889, p. 513-519;

«In Sachen Prostitution und Abolitionismus », Correspondenz-Blatt für Schweizer Ärzte, 22, 1892. p. $654-656$

63. Forel, La question... op. cit., p. 502. 64. Ibid., p. 433. d'éliminer les maladies sexuellement transmissibles, qui représentent avec l'alcool la première cause de mortalité des prostituées et de leurs clients ${ }^{62}$.

\section{LA RÉGLEMENTATION DE LA SEXUALITÉ}

La lutte contre les perversions sexuelles fait partie pour Forel d'un vaste projet d'intervention de l'État et de la classe médicale dans les vies individuelles, qui se compose de deux formes complémentaires : l'eugénisme et la réforme pédagogique. Toute la sexualité humaine, doit s'en tenir à un principe de base : le bien de la société et de l'espèce est toujours plus important que le bien de l'individu. Si la satisfaction de l'appétit sexuel, c'est-à-dire d'un besoin, est reconnue comme un droit de nature, il est tout autant vrai que toutes les actions qui portent atteinte à la liberté ou nuisent à l'existence des autres doivent être interdites et dans certains cas poursuivies pénalement (comme pour la pédophilie héréditaire). La première règle que doit suivre la sexualité humaine consiste alors dans une reformulation originale de l'impératif catégorique kantien : «Par ton appétit et par tes actes sexuels, tu ne nuiras volontairement à aucun individu, ni avant tout à l'humanité, et tu t'efforceras au contraire de favoriser le bonheur de ton prochain et le bien social ${ }^{63}$."

Au nom de ce principe, il faut interdire la procréation aux criminels, aux malades mentaux, aux imbéciles, aux pervers, aux amoraux, aux invalides, aux tuberculeux, aux rachitiques, aux hémophiles et à tous ceux qui sont affectés de maladies héréditaires ou d'une mauvaise constitution. Pour ce faire, on peut recourir même à la stérilisation forcée dans le cas de sujets dangereux et incurables. Une méthode que Forel admet avoir utilisée à au moins deux occasions au Burghölzli, à partir de 1885. Dans La question sexuelle, il raconte comment il a stérilisé, en pratiquant la ligature des trompes, une jeune hystérique de 14 ans dont la mère et la grand-mère avaient été prostituées avant elle. Les troubles de la malade, poursuit Forel, ne sont qu'un simple " prétexte " pour éviter qu'elle puisse se reproduire. Le même sort est appliqué à un "véritable monstre atteint d'anomalies mentales constitutionnelles ${ }^{64}$ ", au prétexte qu'il se plaint de douleurs fréquentes aux organes génitaux.

Ces méthodes eugénistes coercitives sont bien plus efficaces que le néomalthusianisme, c'est-à-dire le contrôle volontaire des naissances (n'oublions pas que le même Forel eut 6 enfants), qui met l'accent sur la nécessité d'une régulation rationnelle de l'augmentation de la population mais qui a le grave 
défaut de se concentrer seulement sur les aspects quantitatifs de la descendance et non sur les aspects qualitatifs ${ }^{65}$. Il faut aussi rejeter l'avortement puisque la mère ou le père n'ont pas le droit de supprimer l'embryon si sa conception est le fruit d'un choix conscient et volontaire. L'interruption doit être permise seulement pour des raisons supérieures d'ordre hygiénique : quand la grossesse est la conséquence d'un viol; quand la santé de la mère ou de l'enfant sont en danger; quand un aliéné rend une femme enceinte; quand une femme idiote ou épileptique tombe enceinte; enfin quand un homme ivre rend enceinte sa femme contre la volonté de cette dernière. Dans le cas de nouveau-nés invalides, difformes ou idiots, on pourrait envisager de recourir, après un diagnostic approprié, à une " douce narcose ${ }^{66}$ ". La conviction que c'est un devoir de les maintenir en vie est la conséquence d'une fausse humanité, déterminée par l'ignorance et par les formes séculières du dogmatisme religieux.

À côté de l'eugénisme, Forel souhaite une rénovation des systèmes éducatifs qui s'inspire aussi bien des principes pédagogiques de Rousseau et de Pestalozzi que de l'organisation des Landerziehungsheime, ces instituts de campagne fondés à partir de 1898 par l'Allemand Hermann Litz. La nouvelle école imaginée par Forel vise au développement intégral de toutes les facultés de l'enfant et elle est basée sur le contact direct avec la nature, sur la mise au travail manuel, sur l'abstinence d'alcool et de drogues, et sur l'éducation sexuelle. Cette dernière doit concerner garçons et filles et empêcher l'apparition de formes excessives de pruderie grâce à des classes mixtes, à l'habitude de la vie en commun et à l'interdiction de lectures pornographiques. Les enfants doivent être encouragés à connaître leur propre corps et les mécanismes et buts des diverses fonctions physiologiques, sexualité comprise. Le mystère qui entoure les questions sexuelles est délétère, non seulement parce qu'il cause angoisse et préoccupation mais également parce qu'il excite morbidement la curiosité des plus jeunes, les poussant à trouver des réponses auprès de leurs camarades plus âgés, dépravés ou dans des lectures pornographiques. Comme les perversions sont dans la plupart des cas innées, il faut un contrôle attentif des maîtres sur les premières manifestations de la sexualité et sur leur régulation. Une fois reconnus, par exemple, les appétits homosexuels, il ne faut pas considérer l'enfant qui les exprime comme un criminel ou un individu mauvais mais comme un "malade atteint d'une affection nerveuse ${ }^{67}$ ". La préoccupation principale vise à empêcher qu'il ne devienne un "foyer d'infection " pour ses camarades, en mettant en

\author{
65. Forel Auguste, \\ Malthusianismus \\ oder Eugenik? 1950 \\ [Malthusianisme ou \\ eugénique?], München, \\ Reinhardt, 1911. Cf. \\ anche « Zur Heilung der \\ Hysterie durch Castration », \\ Correspondenz-Blatt für \\ Schweizer Ärzte, 16, 1886 , \\ p. $476-480$. \\ 66. Forel, La question..., \\ op. cit., p. 454. \\ 67. Ibid., p. 543
}


68. ForeL, La question..., op. cit., p. 546.

69. Ibid., p. 513.

70. Ibid., p. 521.

œuvre une étroite surveillance jour et nuit. Pour éviter l'apparition de pulsions sadiques, les punitions corporelles devront être condamnées, en tant qu'elles sont une " brutalité à la fois inutile et directement nuisible », une « honte pour notre civilisation ${ }^{68} "$.

En conclusion, à la base de ce programme de lutte contre les perversions sexuelles, il y a un socialisme de type " intégral » et " moral », qui voit dans le travail l'instrument privilégié de la libération de l'homme. Pour Forel, il est nécessaire avant tout de bannir définitivement les conflits, qui produisent une sélection « cacogénique » en éliminant les meilleurs éléments de la population et en maintenant en vie les pires, et d'opérer un désarmement progressif des États jusqu'à atteindre une totale disparition des armées. À la place du service militaire, les jeunes gens entre 18 et 19 ans devront contribuer à un « service civil » : Forel suggère que les garçons soient employés dans la construction d'ouvrages publics et dans l'exécution des travaux agricoles les plus lourds, tandis que les filles seraient recrutées comme infirmières dans les hôpitaux. Cette " armée pacifique » se présente comme une école de vie, dans la mesure où elle inculque aux jeunes gens les valeurs de discipline et de résistance à la fatigue, et encourage une "saine camaraderie ", sans distinction de classe.

Les peuples dont on a "scientifiquement » démontré l'infériorité physique et psychique, et l'incapacité à s'élever à un stade civilisé, comme les "nègres " d'Afrique et les indigènes d'Amérique latine et d'Australie, doivent par contre jouir d'une liberté limitée et être mis sous la tutelle des pays occidentaux. La circulation des personnes doit être garantie, exception faite de raisons supérieures d'ordre eugéniste, qui veulent que les individus tarés, invalides et dégénérés, les individus appartenant à des groupes ethniques inférieurs ou racialement trop différents de ceux d'arrivée ne peuvent émigrer parce qu'ils entraîneraient la détérioration physique et psychologique des pays d'accueil.

Au sein d'un projet politique global et articulé, la sexualité juvénile prend donc la forme d'un problème d' "économie politique " et comme tel il doit être affrontét ${ }^{69}$. Le socialisme ne peut ni l'ignorer ni le sous-estimer : il ne peut se limiter à examiner les conditions socio-économiques de la société ou les rapports de production, c'est-à-dire qu'il ne peut transformer la question sociale en une simple « question d'estomac " mais il doit tenir compte des données psychologiques et biologiques et de "l'immixtion perfide des passions sexuelles dans la vie sociale ${ }^{70}$ ». La sexualité est donc la clé de voûte de tous les rapports de 
force et de pouvoir, en bref des rapports entre classes et entre individus : sans la compréhension de la sexualité, on ne peut pas comprendre la société et on ne peut réaliser cet internationalisme socialiste souhaité par Forel. Dans son œuvre se retrouvent ainsi la plupart des discours et des pratiques qui traversent la sexologie et la psychiatrie occidentale entre la seconde moitié du XIX et le début du $\mathrm{xx}^{\mathrm{e}}$ siècle : l'attention portée aux enfants et aux adolescents considérés comme de nouveaux objets de l'intervention psychiatrique non seulement en tant que fous ou idiots, mais aussi parce que sexuellement actifs ${ }^{71}$; l'étude des perversions sexuelles et la tentative de s'affranchir d'une explication organique en faveur d'une vision fonctionnelle; la nouvelle dénomination de perversions déjà partiellement connues, l'intérêt pour le tout nouvel eugénisme anglo-saxon. Dans certains de ces domaines, la réflexion de Forel semble se conformer à l'esprit du temps : dans d'autres, elle présente des éléments d'une indubitable originalité, comme sa considération par moments bienveillante de la masturbation et de l'homosexualité, son soutien aux mouvements pour l'émancipation féminine et surtout sa tentative de lier analyse sociopolitique, question sexuelle et contrôle de la population réalisé via des moyens biopolitiques invasifs, comme l'empêchement de la reproduction voire l'euthanasie des nouveau-nés. Forel est, entre autres, l'exemple emblématique de la transversalité du mouvement eugéniste international depuis ses débuts et de sa capacité à rassembler des savants de milieux culturels et politiques différents. On pouvait être socialiste, antimilitariste, pacifiste, partisan de la reconnaissance des droits des femmes et du respect à l'égard de leur corps, critique des systèmes asilaires de coercition et en même temps se battre pour la stérilisation forcée des malades mentaux et la mise à mort miséricordieuse des enfants difformes. En bref on pouvait être progressiste et eugéniste. Forel fut les deux.

Reste un pessimisme de fond qui parcourt ses écrits. Si beaucoup de perversions sexuelles sont de nature héréditaire, on ne peut pas espérer une régénération complète de la société, malgré l'usage à grande échelle de moyens de contrôle et de répression. Si l'autosuggestion et l'éducation s'avèrent d'une utilité limitée, alors la perversion ne peut être éradiquée complètement. Les enfants affectés d'anomalies sexuelles et psychiques sont destinés à devenir des individus anormaux; au mieux, avec le temps, ils peuvent apprendre à gérer et discipliner leurs propres pulsions ou ils sont rendus, dans la mesure du possible, inoffensifs, éloignés de la menace de l'alcool et de la prostitution. Autrement
71. Outre Les anormaux, cf. aussi Foucault Michel, Le pouvoir psychiatrique. Cours au Collège de France 19731974, Paris, Seuil/Gallimard, 2003, 399 p. 
ambivalente apparaît sa conception de la famille : dans le respect d'un rigide partage des rôles entre mari et femme, celle-ci continue d'être la colonne vertébrale de la société, mais en même temps elle est le lieu par excellence où se commettent abus et violences, soit entre les adultes, soit, de façon plus dramatique, entre les adultes et les enfants. On a l'impression que pour Forel, l'unique façon de protéger les plus petits de la corruption familiale, c'est la méthode décrite par Platon dans La République, à savoir la séparation forcée des enfants d'avec leurs parents. Pour Forel, la sexualité finit donc par être complètement réabsorbée dans le domaine de l'eugénisme. Le sexologue, et plus généralement le médecin, seront eugénistes ou ne seront pas. 